\title{
Classical interpretation of the Debye law for the specific heat of solids
}

\author{
R. Blanco, H. M. França, ${ }^{*}$ and E. Santos \\ Departamento de Física Moderna, Universidad de Cantabria, Santander 39005, Spain
}

(Received 18 July 1990)

\begin{abstract}
We derive the Debye law for the specific heat of solids within the realm of stochastic electrodynamics (i.e., classical electrodynamics with the assumption of a real zero-point field). Random lattice vibrations are generated by the Planck radiation including zero point, which is absorbed by the ions. The equilibrium is accomplished by a fluctuation-dissipation mechanism due to the emission of radiation by the ions in accelerated motion.
\end{abstract}

\section{INTRODUCTION}

The quantum theory of the specific heat of solids, initiated by Einstein ${ }^{1}$ in 1907 and developed later by Debye, ${ }^{2}$ was historically the third large success of quantum theory, after the interpretation of the blackbody spectrum and the photoelectric effect. It is usually considered a typically quantum phenomenon that cannot be explained by any classical theory. ${ }^{3}$ It is therefore interesting to show that this is not the case, by developing a classical theory which reproduces the quantum predictions. The framework of the present approach is stochastic electrodynamics, a theory for the interaction of electromagnetic radiation and charged particles developed in the last 30 years. Stochastic electrodynamics ${ }^{4-6}$ is just classical electrodynamics with the assumption of a real random zero-point radiation filling the whole space, this radiation having precisely the spectrum of the zero-point fluctuating field of quantum electrodynamics, i.e., an energy $\frac{1}{2} \hbar \omega$ per normal mode of the electromagnetic field with frequency $\omega$.

With this assumption one can show that the spectral distribution of the electromagnetic zero-point radiation energy is such that

$$
\rho_{0}(\omega)=\hbar \omega^{3} / 2 \pi^{2} c^{3},
$$

where $c$ is the velocity of light and Planck's constant $2 \pi \hbar$ is the parameter which gives the intensity of this background radiation. It is easy to show that $\rho_{0}(\omega)$ has the interesting property of being Lorentz invariant. ${ }^{4,7}$

The origin of this classical fluctuating zero-point radiation is not very clear yet. However, there are physically appealing propositions which regard the zero-point electromagnetic fields as the radiation emitted by the accelerated charges existing in the matter that fills the universe. ${ }^{8,9}$

In this way stochastic electrodynamics is able to explain, within an entirely classical context, many phenomena considered to belong to the exclusive domain of quantum theory. ${ }^{4-6}$ As examples we have the blackbody radiation, ${ }^{10,11}$ the microscopic properties of the harmonic oscillator, ${ }^{12,13}$ the diamagnetic behavior of free and harmonically bound charges, ${ }^{12,14}$ the paramagnetic behavior of a rigid magnetic dipole, ${ }^{15}$ the Casimir forces between macroscopic objects, ${ }^{16,17}$ and also other phenomena. ${ }^{18}$ As far as we know nobody has been able to obtain the spectrum of the hydrogen atom within the realm of stochastic electrodynamics. There are indications that the ground state of atoms could be considered classically stable, due to the equilibrium ${ }^{4,19}$ between the energy emitted by the accelerated electron and the energy absorbed from the zero-point electromagnetic fields. However, the calculations performed until now do not lead to a stable stationary state of the hydrogen atom. ${ }^{20,21}$

We want to show here that the random zero-point and thermal radiation of stochastic electrodynamics are exactly what is needed to obtain the specific heat of solids within the realm of such a classical theory. The idea is very simple to understand. At temperature $T$ the spectral distribution (1.1) must be replaced by ${ }^{5,6}$

$$
\rho_{T}(\omega)=\frac{\hbar \omega^{3}}{\pi^{2} c^{3}}\left(\frac{1}{2}+\frac{1}{\exp (\hbar \omega / k T)-1}\right),
$$

where $k$ is the Boltzmann constant. Of course the ions in the lattice of the solid body have some kind of classical Brownian motion due to the influence of the random electromagnetic fields. Therefore, the thermal energy of the solid body is simply the mechanical energy associated with that classical Brownian motion. Then the specific heat will be easily obtained from the mechanical energy of the vibrating ions in the lattice.

In order to achieve this goal our paper is arranged as follows. In Sec. II we review the quantum theory of the specific heat of solids in a form appropriate for our purposes. Section III is devoted to a presentation of our classical model. Hence, the proof of our main result is made in Sec. IV. Finally we discuss the results obtained and point out perspectives for future work.

\section{REVIEW OF THE QUANTUM THEORY}

For not too high (and also not extremely low) temperatures, the specific heat of a solid can be associated with the motion of nuclei, the electrons giving a negligible contribution. A more physical picture is to consider ions, instead of bare nuclei, in view of the fact that the inner electrons follow the motions of the nuclei quite closely. If there are $N$ atoms in the solid body, we need $3 N$ coor- 
dinates $x_{i}$ to describe the motion. However, we are allowed to consider only small displacements $\xi_{i}$ around the position of equilibrium $a_{i}$, so that the classical equation of motion for the coordinate $x_{i}=a_{i}+\xi_{i}$ can be written

$$
m \dot{\xi}_{i}=-\sum_{i=1}^{3 N} K_{i j} \xi_{j},
$$

where we assume that $\xi_{i}=0$ corresponds to the position of equilibrium. In practice, all constants $K_{i j}$ are zero except for coordinates of neighbor ions. We have assumed that the ions have the same mass $m$. If the masses are not equal, the change of variables

$$
\left(m_{i} / m\right)^{1 / 2} \xi_{i}=\xi_{i}^{\prime}, \quad m k_{i j} /\left(m_{i} m_{j}\right)^{1 / 2}=k_{i j}^{\prime}
$$

leads again to Eq. (2.1).

In general, ${ }^{22}$ it is possible to perform a coordinate transformation

$$
Q_{l}=\sum_{j=1}^{3 N} A_{l j} \xi_{j}
$$

giving a decoupled set of equations of motion of the form

$$
\ddot{Q}_{l}=-\omega_{l}^{2} Q_{l} \text {, }
$$

where $\omega_{l}$ are the frequencies of the normal modes of vibration. The equations of motion (2.1) can be derived from the Hamiltonian

$$
H=\sum_{j=1}^{3 N}\left[\frac{p_{j}^{2}}{2 m}+\frac{1}{2} \sum_{i=1}^{3 N} K_{i j} \xi_{i} \xi_{j}\right] .
$$

In terms of the new coordinates $Q_{l}$ and the canonical conjugate momenta $P_{l}$, the Hamiltonian can be written

$$
H=\sum_{l=1}^{3 N}\left(\frac{P_{l}^{2}}{2 m}+\frac{1}{2} m \omega_{l}^{2} Q_{l}^{2}\right) .
$$

In quantum mechanics, the starting point is a Hamiltonian operator with the same form as (2.5), except that the coordinates and momenta are operators fulfilling the usual commutation rules.

The Hamiltonian (2.5) shows that the set of ions in the solid body can be treated formally as a set of independent harmonic oscillators. If these oscillators are in thermal equilibrium with a bath at a temperature $T$, each one possesses an average energy

$$
\mathscr{E}_{i}=\frac{1}{2} \hbar \omega_{i} \operatorname{coth}\left(\hbar \omega_{i} / 2 k T\right)
$$

Therefore the total energy will be

$$
\mathscr{E}=\frac{1}{2} \sum_{i=1}^{3 N} \hbar \omega_{i} \operatorname{coth}\left(\hbar \omega_{i} / 2 k T\right)
$$

whence the specific heat can be obtained by

$$
C=\frac{d \mathscr{E}}{d T}
$$

This gives the general expression for the specific heat of a solid body. The particular form depends on the normal-mode frequencies $\omega_{i}$, which can be obtained from the $K_{i j}$ entering in the equation of motion (2.1). In turn, the force constants $K_{i j}$ depend on the lattice structure of the body and the nature of the binding between ions. A good approximation to the sum (2.7) is usually obtained by an integral in the form ${ }^{3}$

$$
\sum_{j=1}^{3 N} \rightarrow \frac{V}{2 \pi^{2}}\left[\frac{1}{v_{l}^{2}}+\frac{2}{v_{t}^{3}}\right] \int_{0}^{k \Theta / \hbar} d \omega \omega^{2},
$$

where $V$ is the volume of the solid, $v_{l}\left(v_{t}\right)$ the velocity of longitudinal (transverse) waves of sound, and $\Theta$ the Debye temperature. This replacement, which is a completely classical procedure, has the advantage that it gives a universal expression for the specific heat as a function of $T / \Theta$.

\section{SOLID BODY IMMERSED IN BLACKBODY RADIATION}

We shall now consider a solid body which has a given lattice structure consisting of a set of pointlike ions with the charges $e_{s}$ and the same mass $m$, immersed in a random electromagnetic radiation having the Planck spectrum (1.2). If the masses are different, the change of variables introduced after Eq. (2.1) leads to a system of equal masses and new charges $\left(m / m_{s}\right)^{1 / 2} e_{s}=e_{s}^{\prime}$. We think that this model is appropriate for ionic solids, where we can use the dielectric constant (and therefore the velocity of light) of vacuum. However, in other types of solids, the electrons not tightly bound to nuclei should be explicitly taken into account, and our model is not adequate.

We shall show that the mechanical energy of the ions is given by the same expression as in the quantum theory, namely, Eq. (2.7), plus corrections of order $e^{2} / \hbar c \approx \frac{1}{137}$. The classical equation of motion for the component $x_{i}(t)$ $(i=1,2 \ldots 3 N)$, associated with the vector position $\mathbf{x}_{s}(t)=\mathbf{a}_{s}+\xi_{s}(t)$ of the ion $s$, immersed in random radiation is

$$
m \ddot{\xi}_{i}(t)=-\sum_{j=1}^{3 N} K_{i j} \xi_{j}(t)+F_{i}^{R}+e_{s} E_{\alpha}\left(\mathbf{a}_{s}, t\right),
$$

where the vectors $\mathbf{a}_{s}$ denote the equilibrium positions of the ions in the lattice $(s=1,2 \ldots . N)$. We call $E_{\alpha}\left(\mathbf{a}_{s}, t\right)$ the $\alpha$ component $(\alpha=0,1,2)$, with respect to three orthogonal axes, of the random electric field in the equilibrium position $\mathbf{a}_{s}$ of the ion $s$. In this study $i=3 s-\alpha$.

Three forces are considered in Eq. (3.1). The first two represent the purely electromagnetic interaction with all the other charges in the solid. The first term consists of the instantaneous Coulomb force, plus the effect of the electronic clouds. The second term, $F_{i}^{R}$, includes the radiation reaction self-force plus the retarded force produced by the other ions. Both are written in the approximation of small displacements around the equilibrium positions. An explicit expression for $F_{i}^{R}$ will be given below in the case of nonrelativistic motion. The last term in (3.1) is the random force on ion $s$ produced by the fluctuating electric field of zero point and thermal radiation. We have neglected the magnetic force and also the 
dependence of the electric field on the small displacement $\xi_{s}$ (but we keep the dependence on $\mathbf{a}_{s}$ ). This is known in the literature as the dipole approximation and it is well known that it is consistent with nonrelativistic motion.

Our purpose is to calculate the average total energy of the ions, which we may write as

$$
\mathscr{E}=\frac{1}{2} \sum_{i=1}^{3 N} m\left\langle\dot{\xi}_{i}^{2}(t)\right\rangle+\frac{1}{2} \sum_{i, j=1}^{3 N} K_{i j}\left\langle\xi_{i}(t) \xi_{j}(t)\right\rangle
$$

where \langle\rangle means both time and ensemble average (or either one if we assume ergodicity). The exact calculation of (3.2) starting from (3.1) is very complex. Fortunately a good approximation can be obtained taking the limit of small charges $\left(e^{2} / \hbar c<1\right)$, and this calculation is rather simple.

To obtain the above mentioned-approximate solution of (3.1), we need the expression for the force $\mathbf{F}_{s}^{R}$ which appears in that equation. As we have already said this force has the contribution of radiation reaction and also the delay effect of the fields of the other charges on the ion $s$. Then we are going to denote

$$
\mathbf{F}_{s}^{R}=\mathbf{F}_{s}(\mathrm{acc})+\mathbf{F}_{s}(\mathrm{vel})
$$

where $\mathbf{F}_{s}(\mathbf{a c c})$ is the contribution from the "acceleration electric fields" and $\mathbf{F}_{s}$ (vel) is the contribution from the "velocity electric fields" of the other ions. The explicit nonrelativistic expression for $\mathbf{F}_{s}$ (acc) is ${ }^{23}$

$$
\begin{aligned}
\mathbf{F}_{s}(\operatorname{acc})= & \frac{2}{3} \frac{e_{s}^{2}}{c^{3}} \ddot{\mathbf{v}}_{s}(t) \\
& +\frac{e_{s}}{c^{2}} \sum_{s^{\prime}(\neq s)} e_{s^{\prime}} \frac{\widehat{\mathbf{n}}_{s s^{\prime}} \times\left[\widehat{\mathbf{n}}_{s s^{\prime}} \times \dot{\mathbf{v}}_{s^{\prime}}\left(t-\tau_{s s^{\prime}}\right)\right]}{\left|\mathbf{a}_{s}-\mathbf{a}_{s^{\prime}}\right|},
\end{aligned}
$$

where

$$
\widehat{\mathbf{n}}_{s s^{\prime}}=\left(\mathbf{a}_{s}-\mathbf{a}_{s^{\prime}}\right) /\left|\mathbf{a}_{s}-\mathbf{a}_{s^{\prime}}\right|,
$$

and

$$
\dot{\mathbf{v}}_{s^{\prime}}\left(t-\tau_{s s^{\prime}}\right)=\ddot{\xi}_{s^{\prime}}\left(t-\tau_{s s^{\prime}}\right)
$$

is the acceleration of ion $s^{\prime}$ in the retarded time $t-\left|\mathbf{a}_{s}-\mathbf{a}_{s^{\prime}}\right| / c$. The first term in (3.4) is the usual nonrelativistic expression for the radiation reaction or "self"force. The second term has the contribution of the other ions. Also well known is the nonrelativistic expression for $\mathbf{F}_{s}$ (vel.). ${ }^{23}$ In the small displacement approximation we get

$\mathbf{F}_{s}(\mathrm{vel})=\sum_{s^{\prime}(\neq s)} \frac{e_{s} e_{s^{\prime}}}{\left|\overline{\mathbf{a}}_{s}-\overline{\mathbf{a}}_{s^{\prime}}\right|^{2}}\left[\frac{\xi_{s^{\prime}}(t)-\boldsymbol{\xi}_{s^{\prime}}\left(t-\tau_{s s^{\prime}}\right)}{\left|\mathbf{a}_{s}-\mathbf{a}_{s^{\prime}}\right|}-3 \widehat{\mathbf{n}}_{s s^{\prime}} \frac{\boldsymbol{\xi}_{s^{\prime}}(t)-\boldsymbol{\xi}_{s^{\prime}}\left(t-\tau_{s s^{\prime}}\right)}{\left|\mathbf{a}_{s}-\mathbf{a}_{s^{\prime}}\right|} \widehat{\mathbf{n}}_{s s^{\prime}}+3 \widehat{\mathbf{n}}_{s s^{\prime}} \frac{\hat{\mathbf{n}}_{s s^{\prime}} \cdot \mathbf{v}_{s^{\prime}}\left(t-\tau_{s s^{\prime}}\right)}{c}-\frac{\mathbf{v}_{s^{\prime}}\left(t-\tau_{s s^{\prime}}\right)}{c}\right]$,

where we are using the same notation as in (3.4), that is, $\mathbf{v}_{s^{\prime}}\left(t-\tau_{s s^{\prime}}\right)=\dot{\boldsymbol{\xi}}_{s^{\prime}}\left(t-\tau_{s s^{\prime}}\right)$ with $\tau_{s s^{\prime}}=\left|\mathbf{a}_{s}-\mathbf{a}_{s^{\prime}}\right| / c$.

In Eq. (3.5) the instantaneous Coulomb force has been removed because it is supposed to be included in the first term of Eq. (3.1). The first and the second terms in (3.5) are simply corrections to the Coulomb force due to small $\left(\left|\xi_{s}-\xi_{s^{\prime}}\right| /\left|\mathbf{a}_{s}-\mathbf{a}_{s^{\prime}}\right|<1\right)$ displacements from the equilibrium positions. The retardation is, of course, due to the fact that electromagnetic signals travel with finite velocity $c$. The last two terms in (3.5) are simply retarded relativistic corrections to the velocity electric fields. Terms of order $v^{2} / c^{2}$ have been neglected. We want to stress that each of the above-mentioned terms, which contributes to $\mathbf{F}_{s}^{R}$, has a precise role in the dynamic equilibrium, which is supposed to give the stationary regime in which the average solid energy is given by (3.2). We are going to explain this point in detail in Sec. IV.

The most important ingredient of our model is the random force $e_{s} \mathbf{E}\left(\mathbf{a}_{s}, t\right)$, which is present in the equation of motion (3.1). This force is generated by the fluctuating electric field of zero point and thermal radiation, characteristic of stochastic electrodynamics. Within this classical theory it is usual to write the electric field, in point $\mathbf{x}$ at time $t$, as a superposition of plane waves with frequencies $\omega=c|\mathbf{k}|$ where $\mathbf{k}$ is the wave vector. Namely, we have $\mathrm{e}^{24}$

$$
\begin{aligned}
E_{\alpha}(\mathbf{x}, t)=\sum_{\lambda=1}^{2} \int & d^{2} k \hat{\epsilon}_{\alpha}(\mathbf{k}, \lambda)\left[\frac{\mathrm{c}^{3} \rho_{\mathrm{T}}(\omega)}{4 \omega^{2}}\right]^{1 / 2} \\
& \times\{c(\mathbf{k}, \lambda) \exp [i(\mathbf{k} \cdot \mathbf{x}-\omega t)]+\text { c.c. }\}
\end{aligned}
$$

where $c(\mathbf{k}, \lambda)$ are statistically independent Gaussian complex random variables with the two first moments given by

$$
\begin{aligned}
& \langle c(\mathbf{k}, \lambda)\rangle=0, \quad\left\langle c(\mathbf{k}, \lambda) c\left(\mathbf{k}^{\prime}, \lambda^{\prime}\right)\right\rangle=0, \\
& \left\langle c(\mathbf{k}, \lambda) c^{*}\left(\mathbf{k}^{\prime}, \lambda^{\prime}\right)\right\rangle=\delta\left(\mathbf{k}-\mathbf{k}^{\prime}\right) \delta_{\lambda \lambda^{\prime}},
\end{aligned}
$$

$\rho_{T}(\omega)$ is the spectral distribution (1.2) and the unit vectors $\hat{\boldsymbol{\epsilon}}(\mathbf{k}, \lambda)$ give the transverse polarization of the waves.

Now we can have a precise idea of our model. It is obvious that, despite the fact that $\mathrm{Eq}$. (3.1) is linear on the variables $\xi_{i}(t)$, the exact solution of (3.1) is not trivial. Therefore, we are going to find a stationary solution for (3.1) which is valid up to first order in $e^{2} / \hbar c=\frac{1}{137}$.

Before we pass to the discussion of the abovementioned stationary solution, it is convenient to display a useful property of the random fields (3.6). We shall use this property in order to obtain the average energy (3.2). From (3.6) and (3.7) we can obtain the correlation function between the components $E_{\alpha}(\mathbf{x}, t)$ and $E_{\beta}\left(\mathbf{x}^{\prime}, t\right)$ at ar- 
bitrary separation $\mathbf{x}-\mathbf{x}^{\prime}$. This can be calculated using the standard procedure explained before by many authors. ${ }^{4-6}$ In the case in which $\mathbf{x}$ and $\mathbf{x}^{\prime}$ are two arbitrary points $\mathbf{a}_{s}$ and $\mathbf{a}_{s^{\prime}}$ in the solid lattice we get

$$
\begin{aligned}
e_{s} e_{s^{\prime}}\left\langle E_{\alpha}\left(\mathbf{a}_{s}, t\right) E_{\beta}\left(\mathbf{a}_{s^{\prime}}, t\right)\right\rangle \\
=\int_{0}^{\infty} d \omega \rho_{T}(\omega) B_{i j}\left(\omega\left|\mathbf{a}_{s}-\mathbf{a}_{s^{\prime}}\right| / c\right),
\end{aligned}
$$

where $i=3 s-\alpha, j=3 s^{\prime}-\beta$, and $E_{\alpha}$ are the three Cartesian components of the electric field. The matrix $B_{i j}$ is such that any $3 \times 3$ submatrix corresponding to fixed values of $s$ and $s^{\prime}$ can be obtained by a straightforward rotation of the diagonal matrix

$$
\begin{aligned}
& b_{x x}(u)=b_{y y}(u)=4 \pi e_{s} e_{s^{\prime}}\left(\frac{\sin u}{u}+\frac{\cos u}{u^{2}}-\frac{\sin u}{u^{3}}\right), \\
& b_{z z}(u)=8 \pi e_{s} e_{s^{\prime}}\left(\frac{\sin u}{u^{3}}-\frac{\cos u}{u^{2}}\right), \\
& u \equiv \omega\left|\mathbf{a}_{s}-\mathbf{a}_{s^{\prime}}\right| / c,
\end{aligned}
$$

where now the direction labeled $z$ is parallel to $\mathbf{a}_{s}-\mathbf{a}_{s^{\prime}}$ and the directions $x$ and $y$ are perpendicular to each other and to $z$. [For $u=0$ take $u \rightarrow 0$ in (3.9).]

We shall see below how the correlation function (3.8) combines itself with the contribution of the force $\mathbf{F}_{s}^{R}$ (3.3) to the average solid energy (3.2), in order to preserve the fluctuation-dissipation relation so common to classical theories of Brownian motion.

\section{TOTAL AVERAGE SOLID-BODY ENERGY}

The first step to obtain the energy of the whole set of coupled oscillators is to solve the equation of motion (3.1). One can do this by using a Fourier transform defined by

$$
\widetilde{\xi}_{j}(\omega)=\frac{1}{2 \pi} \int_{-\infty}^{\infty} d t \xi_{j}(t) e^{i \omega t} .
$$

Hence the equation of motion (3.1) becomes

$$
-m \omega^{2} \widetilde{\xi}_{i}(\omega)=-\sum_{j=1}^{3 N} K_{i j} \widetilde{\xi}_{j}(\omega)+\widetilde{F}_{i}^{R}+e_{i} \widetilde{E}_{i}(\omega) .
$$

For consistency in the notation we write from now on $e_{i}$ instead of $e_{s}$; see discussion following Eq. (3.1).

In (4.2) the Fourier transform of the force $F_{i}^{R}$ is

$$
\widetilde{F}_{i}^{R}(\omega)=\frac{1}{2 \pi} \int_{-\infty}^{\infty} d t F_{i}^{R}(t) e^{i \omega t}
$$

and the Fourier transform of the $\alpha$ component of the electric field is

$$
\widetilde{E}_{i}(\omega)=\frac{1}{2 \pi} \int_{-\infty}^{\infty} d t E_{\alpha}\left(\mathbf{a}_{s}, t\right) e^{i \omega t}
$$

where, as before, $i=3 s-\alpha, s=1,2, \ldots, N$, and $\alpha=0,1,2$.

It is convenient to introduce a matrix $\underline{R}(\omega)$ whose elements $R_{i j}(\omega)$ are defined as

$$
\widetilde{F}_{i}^{R}(\omega)=-\sum_{j=1}^{3 N} R_{i j}(\omega) \widetilde{\xi}_{j}(\omega)
$$

The exact expression for the elements $R_{i j}(\omega)$ will be presented below. In terms of the matrix elements $R_{i j}(\omega)$ the equation of motion becomes

$$
\sum_{j=1}^{3 N}\left[K_{i j}+R_{i j}(\omega)-m \omega^{2} \delta_{i j}\right] \widetilde{\xi}_{j}(\omega)=e_{i} \widetilde{E}_{i}(\omega),
$$

which has the exact solution

$$
\widetilde{\xi}_{i}(\omega)=\sum_{j=1}^{3 N}\left[\underline{L}^{-1}(\omega)\right]_{i j} \widetilde{E}_{j}(\omega) e_{j},
$$

in terms of the inverse of the matrix

$$
\underline{L}(\omega)=\underline{K}+\underline{R}(\omega)-m \omega^{2} \underline{I},
$$

where $\underline{I}$ is the identity matrix $(\underline{I})_{i j}=\delta_{i j}$ and $(\underline{K})_{i j}=K_{i j}$. Since we have the exact solution (4.7) the ensemble average total energy, namely,

$$
\mathscr{E}=\frac{1}{2} m \sum_{i=1}^{3 N}\left\langle\dot{\xi}_{i}^{2}(t)\right\rangle+\frac{1}{2} \sum_{i, j=1}^{3 N} K_{i j}\left\langle\xi_{i}(t) \xi_{j}(t)\right\rangle,
$$

can be written in a closed form as

$$
\begin{aligned}
\mathscr{E}=\frac{c^{3}}{2} \int_{0}^{\infty} d \omega \rho_{T}(\omega) \operatorname{Tr}\{ & {\left[\underline{L}^{-1}(\omega)\right]^{\dagger} } \\
& \left.\times\left(m \omega^{2} \underline{I}+\underline{K}\right) \underline{B}(\omega) \underline{L}^{-1}(\omega)\right\},
\end{aligned}
$$

where we have used expression (3.8) for the correlation function of the random electric field between two points $\mathbf{a}_{s}$ and $\mathbf{a}_{s^{\prime}}$ in the lattice.

In order to calculate the total average energy by integrating (4.10) we need the expression for the matrix elements of $\underline{R}(\omega)$ which appears in (4.8) and, consequently, in (4.10). The elements $R_{i j}(\omega)$ can be obtained directly from its definition [(4.5)] in terms of the Fourier transform of the force $\mathbf{F}^{R}$. It has many terms, as one can see from (3.3), (3.4), and (3.5), but the detailed calculation is straightforward. We only give the final result. The elements $R_{i j}(\omega)$ are complex functions of the argument and we can show that the imaginary part is such that

$$
\operatorname{Im}\left[R_{i j}(\omega)\right]=\frac{1}{4 \pi} \omega^{3} B_{i j}(\omega)
$$

where the matrix $\underline{B}(\omega)$ was defined in (3.8). This result will be very useful, as we shall see below. The real part of $R_{i j}(\omega)$ is zero for $s=s^{\prime}$. For different given $s$ and $s^{\prime}$ the corresponding $3 \times 3$ submatrix is related by a rotation to the diagonal matrix,

$$
\begin{aligned}
& r_{x x}=r_{y y}=-\frac{e_{s} e_{s^{\prime}} \omega^{3}}{c^{3} u^{3}}\left(1-\cos u-u \sin u+u^{2} \cos u\right), \\
& r_{z z}=-\frac{e_{s} e_{s^{\prime}} \omega^{3}}{c^{3} u^{3}} 2(\cos u+u \sin u-1), \\
& u=\omega\left|\mathbf{a}_{s}-\mathbf{a}_{s^{\prime}}\right| / c,
\end{aligned}
$$

where the directions $x, y, z$ are as in Eq. (3.9).

In order to obtain the average energy we must calculate the trace indicated in (4.10). For this a diagonaliza- 
tion procedure is convenient, giving, as shown in the Appendix,

$$
\begin{aligned}
\operatorname{Tr}\left[\left(\underline{L}^{-1}\right)^{\dagger}\left(m \omega^{2} \underline{I}+\underline{K}\right) \underline{B} \underline{L}^{-1}\right] \\
\simeq \sum_{j=1}^{3 N} \frac{m\left(\omega^{2}+\widetilde{\omega}_{j}^{2}\right) B_{j j}^{\prime}}{\left[m\left(\widetilde{\omega}_{j}^{2}-\omega^{2}\right)\right]^{2}+\left[\operatorname{Im} R_{j j}^{\prime}\right]^{2}}
\end{aligned}
$$

if we neglect terms of second order in $e^{2} / \hbar c$. Here the matrices $\underline{B}^{\prime} \equiv \underline{\widetilde{A}} \underline{B} \underline{A}$, and $\underline{R}^{\prime} \equiv \underline{\widetilde{A}} \underline{R} \underline{A}$ (see Appendix for the definition of $\underline{A}$ ) are both first order in $e^{2} / \hbar c$, as we can see from (3.8) and (4.11). The frequencies $\widetilde{\omega}_{j}$, which are the eigenvalues of the matrix $\underline{\bar{K}} \equiv \underline{K}+\operatorname{Re}(\underline{R})$, differ from the normal-mode frequencies $\omega_{j}$, eigenvalues of $\underline{K}$, by a negligible radiative correction of order

$$
\operatorname{Re}\left(R_{j j}^{\prime}\right) / m \omega_{j}^{2} \sim\left(e^{2} / \hbar c\right)\left(\hbar \omega_{j} / m c^{2}\right) \ll<,
$$

since we expect $\hbar \omega_{j}$ to be about a few electron volts and the ion masses are such that $m c^{2} \approx 10^{10} \mathrm{eV}$.

The average energy (4.10) becomes in this approximation

$$
\mathscr{E}=\frac{c^{3}}{2} \sum_{j=1}^{3 N} \int_{0}^{\infty} d \omega \frac{m\left(\omega^{2}+\omega_{j}^{2}\right) B_{j j}^{\prime}(\omega) \rho_{T}(\omega)}{\left[m\left(\omega_{j}^{2}-\omega^{2}\right)\right]^{2}+\left(\operatorname{Im} R_{j j}^{\prime}\right)^{2}}
$$

and the integral can be calculated easily as follows. The integrand in (4.14) has very sharp peaks for $\omega$ close to the normal-mode frequencies $\omega_{j}$. This happens because the denominator in (4.14) is very small for frequencies $\omega$ close to $\omega_{j}$ due to the fact that

$$
\operatorname{Im}\left(R_{j j}^{\prime}\right) / m \omega_{j}^{2} \sim\left(e^{2} / \hbar c\right)\left(\hbar \omega_{j} / m c^{2}\right)<<1 .
$$

Therefore the integrals which remain to be performed in (4.16) are essentially like

$$
\int_{-\omega_{j}}^{\infty} d x \frac{1}{x^{2}+\gamma_{j}^{2}} \simeq \frac{\pi}{\gamma_{j}}
$$

since $\gamma_{j} \equiv\left|\operatorname{Im}\left[R_{j j}^{\prime}\left(\omega_{j}\right)\right] / m \omega_{j}\right| \ll \omega_{j}$. Note that the approximation (4.15) is equivalent to taking the limit $e \rightarrow 0$ in (4.14). The resulting expression for the average energy is therefore

$$
\mathscr{E}=\frac{\pi c^{3}}{4} \sum_{j=1}^{3 N} \omega_{j} \rho_{T}\left(\omega_{j}\right) \frac{B_{j j}^{\prime}\left(\omega_{j}\right)}{\left|\operatorname{Im} R_{j j}^{\prime}\left(\omega_{j}\right)\right|}
$$

where the coefficients $B_{j j}^{\prime}$ can be shown to be positive. Moreover, we have

$$
\frac{\left|B_{j j}^{\prime}\left(\omega_{j}\right)\right|}{\left|\operatorname{Im} R_{j j}^{\prime}\left(\omega_{j}\right)\right|}=\frac{\left|\sum_{l, k}(\widetilde{A})_{j l} B_{l k}\left(\omega_{j}\right) A_{k j}\right|}{\left|\sum_{l, k}(\widetilde{A})_{j l}\left[\operatorname{Im} R_{l k}\left(\omega_{j}\right)\right] A_{k j}\right|}=\frac{4 \pi}{\omega_{j}^{3}}
$$

because $\operatorname{Im} R_{i j}$ and $B_{i j}$ obey the simple relation (4.11). Then we finally obtain

$$
\mathscr{E}=\sum_{j=1}^{3 N} \hbar \omega_{j}\left(\frac{1}{2}+\frac{1}{\exp \left(\hbar \omega_{j} / k T\right)-1}\right),
$$

which is precisely the quantum expression (2.7). The specific heat can be obtained along the previous lines of reasoning, namely, (2.8) and (2.9), which are essentially classical.

\section{DISCUSSION}

We have considered a system of classical charged particles subject to forces given by the Hamiltonian (2.4) plus damping and retarded forces as given by (3.4) and (3.5), which includes the self-force of radiation reaction, the radiative effects due to the other charged particles, and also the action of an isotropic random radiation with spectral density given by the Planck formula (1.2). We have shown that this system has a total average energy given by (4.18) where the $\omega_{j}$ are the normal-mode frequencies of the Hamiltonian (2.4). This completes our derivation of the specific heat of solid bodies. The question whether this derivation uses only classical postulates deserves some attention. The answer is clearly affirmative if there exists an independent classical derivation of the Planck spectrum for blackbody radiation, as has been claimed many times. ${ }^{10,25}$ In our opinion, a classical uncontroversial derivation does not exist. ${ }^{1,26,27}$ In spite of this, the present derivation of the Debye specific-heat law, using classical equations of motion for Brownian charged particles, serves to clarify many points.

(i) It provides a classical interpretation of the specific heat as being due to a continuous distribution of energies of the normal-model vibrations. The spectral distribution of these vibrations is given by (1.2). In particular, no assumption of discreteness is needed for the energies of the solid vibrations. The conventional classical equipartition description (leading to the Dulong and Petit law) is obtained only in the high-temperature limit.

(ii) It shows that the close connection (discovered by Einstein in 1907) between the Planck blackbody spectrum and the specific-heat law is maintained even if both are interpreted along classical ideas. This is the main result of our paper.

(iii) It gives another interesting example of the validity of the fluctuation-dissipation relation, in a classical theory of Brownian motion with colored noise. Our result (4.18) is a direct consequence of (4.11) in which the left-hand side is connected with the forces (3.4) and (3.5), and the right-hand side is due to the correlations of the random fields in different points in the lattice. In our opinion this reinforces previous propositions ${ }^{8,9}$ which consider that the charged-particle Brownian motion generates the random electromagnetic radiation, and this in turn provides a source of noise in the form of a selfregenerating feedback cycle.

(iv) It may allow the calculation of the corrections of order $e^{2} / \hbar c$ to the specific-heat law in a simpler manner than the conventional quantum electrodynamics calculation (not yet made to our knowledge).

(v) It may be the starting point for further elaboration of the theory of solids along classical ideas, thus allowing the classical interpretation of other properties.

\section{ACKNOWLEDGMENTS}

This work was partially supported by Dirección General de Investación Científica y Técnica (Spain) under 
Contract No. PB 87-0014. One of us (H.M.F.) acknowledges the hospitality of the Universidad de Cantabria and also the financial support from Fundaçao de Amparo a Pesquisa do Estado de São Paulo (FAPESP), Conselho Nacional de Desenvolvimento Científico e Tecnológico (CNPq), and Programa Banco Interamericano de Desenvolvimento-Universidade de São Paulo.

\section{APPENDIX}

We want to calculate the trace appearing in Eq. (4.10). Since we need to invert the matrix $\underline{L}$, the simplest way consists in diagonalizing this matrix. First of all we write $\underline{L}$ as

$$
\underline{L}=\underline{\bar{K}}+i \operatorname{Im} \underline{R}-m \omega^{2} \underline{I}
$$

with

$$
\underline{\bar{K}}=\underline{K}+\operatorname{Re} \underline{R} .
$$

Since $\underline{K}$ and $\underline{R}$ are symmetric matrices, so is $\underline{K}$. Consequently we can diagonalize it by means of an orthogonal transformation, that is, there exists a matrix $\underline{A}$ fulfilling

$$
\begin{aligned}
& \underline{\widetilde{A}} \underline{\bar{K}} \underline{A}=\underline{\bar{K}}^{\prime} \equiv m \operatorname{diag}\left(\ldots \widetilde{\omega}_{i}^{2} \ldots\right), \\
& \underline{\widetilde{A}} \underline{A}=\underline{A} \underline{\tilde{A}}=\underline{I} .
\end{aligned}
$$

The transformed matrix $\underline{L}^{\prime}$ is given by

$$
\underline{L}^{\prime} \equiv \tilde{A} \underline{L} \underline{A}=\underline{\bar{K}}^{\prime}+i \operatorname{Im} \underline{R}^{\prime}-m \omega^{2} \underline{I}
$$

in terms of the transformed matrix $\underline{R}^{\prime}$

$$
\underline{R}^{\prime} \equiv \underline{\widetilde{A}} \underline{R} \underline{A} \text {. }
$$

These new matrices $\underline{L}^{\prime}$ and $\underline{R}^{\prime}$ are not in general diagonal. However, we can achieve an approximate diagonalization if we only keep terms of order $e^{2}$. To do that let us define the matrix

$$
\underline{M}=\underline{I}+\underline{C}
$$

with

$$
C_{i j}=-i \frac{\operatorname{Im} R_{i j}^{\prime}}{m\left(\widetilde{\omega}_{i}^{2}-\widetilde{\omega}_{j}^{2}\right)}, \quad i \neq j, \quad C_{i i}=0 .
$$

(We assume that all frequencies are different. See below for the case of degeneracy). Since $\underline{R}^{\prime}=O\left(e^{2}\right)$, then

$$
\underline{M}^{-1} \cong \underline{I}-\underline{C} \text {. }
$$

Now, by using (A7), (A8), and (A9), a simple calculation gives

$$
\begin{aligned}
\underline{L}^{\prime \prime} & \equiv \underline{M}^{-1} \underline{L}^{\prime} \underline{M} \\
& =\underline{\bar{K}}^{\prime}-m \omega^{2} \underline{I}+i\left(\operatorname{Im} \underline{R}^{\prime}\right)+\underline{\bar{K}}^{\prime} \underline{C}-\underline{C} \underline{\bar{K}}^{\prime}+O\left(e^{4}\right),
\end{aligned}
$$

whence, for $i \neq j$,

$$
L_{i j}^{\prime \prime}=i\left(\operatorname{Im} R_{i j}^{\prime}\right)+m \omega_{i}^{2} C_{i j}-m C_{i j} \omega_{j}^{2}+O\left(e^{4}\right)
$$

and

$$
L_{i i}^{\prime \prime}=L_{i i}^{\prime}+O\left(e^{4}\right) .
$$

Consequently, the matrix $\underline{A} \underline{M}$ diagonalizes $\underline{L}$ up to order $e^{2}$. Note that the last step indicates that the off-diagonal elements of $\operatorname{Im} \underline{R}^{\prime}$, which are in principle of order $e^{2}$, in fact contribute to the trace with terms of order $e^{4}$.

The calculation of (4.13) can be made much easier after the transformation with $\underline{A} \underline{M}$

$$
\begin{aligned}
\operatorname{Tr}\left[\left(\underline{L}^{-1}\right)^{\dagger}\left(m \omega^{2} \underline{I}+\underline{K}\right) \underline{B} \underline{L}^{-1}\right] \\
\quad=\operatorname{Tr}\left[\left(\underline{L}^{\prime \prime-1}\right)^{\dagger}\left(m \omega^{2} \underline{I}+\underline{K}^{\prime \prime}\right) \underline{B}^{\prime \prime} \underline{L}^{-1}\right] \\
\quad=\sum_{i, j}\left(L_{i i}^{\prime \prime}\right)^{-1}\left(m \omega^{2} \delta_{i j}+K_{i j}^{\prime \prime}\right) B_{j i}^{\prime \prime}\left(L_{i i}^{\prime \prime}\right)^{-1} .
\end{aligned}
$$

As can be seen in the text the matrix $\underline{K}^{\prime}$ differs from $\underline{K}^{\prime \prime}$ in terms that do not contribute to the final expression. This is because we are interested in the terms that are of lowest order in the charges. Consequently we can write (A13) as

$$
\begin{aligned}
\sum_{i}\left(L_{i i}^{\prime \prime}\right)^{-1}\left(m \omega^{2}+\underline{\bar{K}}_{i i}^{\prime}\right) B_{i i}^{\prime \prime}\left(L_{i i}^{\prime \prime}\right)^{-1} \\
=\sum_{i} \frac{m\left(\omega^{2}+\widetilde{\omega}_{i}^{2}\right) B_{i i}^{\prime}}{\left[m\left(\widetilde{\omega}_{i}^{2}-\omega^{2}\right)\right]^{2}+\left(\operatorname{Im} R_{i i}^{\prime}\right)^{2}},
\end{aligned}
$$

which is the desired result. In this expression we have taken into account (A5) and (A12).

If not all the frequencies were different we may diagonalize $\operatorname{Im} \underline{R}^{\prime}$ in each subspace corresponding to equal frequencies, which leaves the matrices $\underline{K}^{\prime}$ and $m \omega^{2} \underline{I}$ invariant. In other words, we could choose the matrix $\underline{A}$ such that $\operatorname{Im} \underline{R}^{\prime}$ has vanishing elements for indices with equal frequencies. Consequently, the expression (A14) remains valid in any case. This ends the proof of Eq. (4.13).
* On leave from Instituto de Física, Universidade de São Paulo, São Paulo, Brazil.

${ }^{1}$ A. Einstein, Ann. Phys. (Leipzig) 22, 180 (1907); 34, 170 (1911).

${ }^{2}$ P. Debye, Ann. Phys. (Leipzig) 39, 789 (1912).

${ }^{3}$ M. Born, Atomic Physics (Hafner, New York, 1961), Chap. 8.

${ }^{4}$ T. H. Boyer, Phys. Rev. D 11, 790 (1975); see also the review paper in Foundations of Radiation Theory and Quantum Electrodynamics, edited by A. Barut (Plenum, New York, 1980), p. 49; Sci. Ann. 293, 56 (1985).

${ }^{5}$ P. W. Milonni, Phys. Rep. 25, 1 (1976).

${ }^{6}$ L. de la Peña, in Stochastic Processes Applied to Physics and
Other Related Fields, edited by B. Gómez, S. M. Moore, A. M. Rodríguez-Vargas, and A. Rueda (World Scientific, Singapore, 1982), p. 428.

${ }^{7}$ T. W. Marshall, Proc. Cambridge Philos. Soc. 61, 537 (1965).

${ }^{8}$ E. Santos, in Proceedings of the Einstein Centennial Symposium on Fundamental Physics, edited by S. M. Moore, A. M. Rodríguez-Vargas, and A. Rueda (Universidad de los Andes, Bogotá, 1979), p. 213.

${ }^{9}$ H. E. Puthoff, Phys. Rev. A 40, 4857 (1989).

${ }^{10}$ T. H. Boyer, Phys. Rev. 182, 1374 (1969); 186, 1304 (1969).

${ }^{11}$ J. L. Jiménez, L. de la Peña, and T. A. Brody, Am. J. Phys. 48, 
840 (1980).

12T. W. Marshall, Proc. R. Soc. London 276, 475 (1963).

${ }^{13}$ E. Santos, Nuovo Cimento B 19, 57 (1974).

${ }^{14}$ T. H. Boyer, Phys. Rev. D 11, 809 (1975).

${ }^{15}$ A. V. Barranco, S. A. Brunini, and H. M. França, Phys. Rev. A 39, 5492 (1989).

${ }^{16}$ T. W. Marshall, Nuovo Cimento 38, 206 (1965).

${ }^{17}$ T. H. Boyer, Ann. Phys. (N.Y.) 56, 474 (1970).

${ }^{18}$ T. W. Marshall and E. Santos, Found. Phys. 18, 185 (1988); Phys. Rev. A 39, 6271 (1989); see also A. V. Barranco and H. M. França, Phys. Essays 3, 53 (1990), for a qualitative discussion of the Compton effect within the realm of classical stochastic electrodynamics.

${ }^{19}$ H. E. Puthoff, Phys. Rev. D 35, 3266 (1987).

${ }^{20}$ T. W. Marshall and P. Claverie, J. Math. Phys. 21, 1819
(1980).

${ }^{21}$ P. Claverie, L. Pesquera, and F. Soto, Phys. Lett. A 80, 113 (1980).

${ }^{22} \mathrm{H}$. Goldstein, Classical Mechanics (Addison-Wesley, New York, 1980), Chap. 6.

${ }^{23}$ J. D. Jackson, Classical Electrodynamics (Wiley, New York, 1975), Chap. 14.

${ }^{24}$ E. Santos, Nuovo Cimento B 22, 201 (1974).

${ }^{25}$ T. H. Boyer, Phys. Rev. D 27, 2906 (1983); 29, 1096 (1984); Found. Phys. 19, 1731 (1989).

${ }^{26}$ R. Blanco, L. Pesquera, and E. Santos, Phys. Rev. D 27, 1254 (1983); 29, 2240 (1984).

${ }^{27}$ H. M. França and G. C. Santos, Nuovo Cimento B 86, 51 (1985). 